im Perjodat-Kollagen zu 0,00164-m., folglich enthält Perjodat-Kollagen noch $0,26 \%$ Oxylysin.

b) Tyrosin: Seine Bestimmung erfolgte nach Fritze und $\mathrm{Z}_{\mathrm{AHN}}{ }^{18}$. Während normales Kollagen $0,71 \%$ Tyrosin aufwies, enthielt Perjodat-Kollagen noch $0,03 \%$, also praktisch kein Tyrosin.

III. Di e P e r jodat-Oxydation von Wolle : $500 \mathrm{mg}$ Wolle, $2,5 \mathrm{~g} \mathrm{NaJO}_{4}$ und $100 \mathrm{mg} \mathrm{NaHCO}_{3}$ wurden in $200 \mathrm{~cm}^{3}$ Wasser aufgeschlämmt und unter $\mathrm{CO}_{2}$ Durchleiten und gutem Rühren auf $80^{\circ} \mathrm{C}$ erwärmt. Nach 2-3 Stdn. war die Wolle fast restlos in Lösung gegangen. Die Beseitigung des Oxydationsmittels, Entsalzung und Gefriertrocknung erfolgte ähnlich wie beim
Kollagen ${ }^{5}$. 50\% der aufgelösten Wolle gingen beim Dialysieren verloren, was durch Stickstoffbestimmung festgestellt wurde. Das nicht dialysable gefriergetrocknete Produkt war gelblich gefärbt.

$100 \mathrm{mg}$ Perjodat-Wolle wurden, wie oben bei Perjodat-Kollagen beschrieben, hydrolysiert und zur Chromatographie vorbereitet. Aus Methionin war Methioninsulfon mit dem $R_{f}$-Wert 0,55 in Phenol/Wasser und 0,1 in sek. Butanol/Ameisensäure/Wasser 75/15/10 entstanden. Die chromatographisch beobachtete Abnahme von Tyrosin und Cystin wurde wie oben bestimmt ${ }^{18,20}$. Beide Aminosäuren waren ebenso wie Tryptophan (bestimmt nach 1. c. ${ }^{19}$ ) praktisch völlig zerstört.

\title{
Über die Kondensation von Formaldehyd mit 2-Acetaminoacetyl-thiophen und $\boldsymbol{\omega}$-Acetamino-acetophenon
}

\author{
Von Otтo Dann und Götz Benedickт * \\ Aus dem Institut für angewandte Chemie der Universität Erlangen \\ (Z. Naturforschg. 12 b, 791-794 [1957]; eingegangen am 11. Juli 1957)
}

\begin{abstract}
Die bei der Anlagerung von $2 \mathrm{HCHO}$ an 2-Acetaminoacetyl-thiophen (I) und $\omega$-Acetamino-acetophenon (II) entstehenden doppelten Kondensationsprodukte V und VI spalten bei der M e e r w e i n P on nd or f - Reduktion HCHO ab.
\end{abstract}

Bei der Anlagerung von Formaldehyd an 2-Acetaminoacetyl-thiophen (I) erhielten sowohl Carrara und Weitnauer ${ }^{1}$ als auch Keskin, Mason und Nord ${ }^{2}$ das bei $113^{\circ}$ schmelzende DL-2-( $\alpha$-Acetamino- $\beta$ hydroxy-propionyl)-thiophen (III), während von $\mathrm{D}_{\text {ANN, UlRich und MöLleR }}{ }^{3}$ ein bei $167^{\circ}$ schmelzendes Kondensationsprodukt $\mathrm{V}$ erhalten wurde mit angeblich gleichem Stickstoffgehalt.

Durch freundliche Privatmitteilung von Herrn Prof. G. Carrara ${ }^{4}$ wurden wir darauf aufmerksam gemacht, daß diese Stickstoffanalyse falsch ist, daß die Verbindung vom Schmp. $167^{\circ}$ sich vielmehr als doppeltes Kondensationsprodukt V analysiert, welches aus 2 Moll. Formaldehyd und 1 Mol. 2-Acetaminoacetyl-thiophen (I) entsteht, und daß unter den von DANN, Ulrich und Möller ${ }^{3}$ angegebenen Versuchsbedingungen fast ausschließlich das bei $113^{\circ}$ einfache Kondensationsprodukt (III) erhalten wird.

* Aus der Dissertation G. Benedickt, Erlangen 1957.

1 G. Carrara u. G. Weitnauer, Gazz. chim. ital. 81, 142. [1951].

2 H. Keskin, C. D. MASon u. F. Nord, J. org. Chemistry 16, 1333 [1951].

3 O. DanN, H. Ulrich u. E. F. Möller, Z. Naturforschg. 7 b, 344 [1952].
Daraufhin haben wir die früheren ${ }^{3}$ Angaben nachgeprüft. Zuerst wurden die Originalpräparate von ULRICH $^{5}$ nachanalysiert; es bestätigten sich die Analysenergebnisse von Eтtore und $\mathrm{VecchI}^{4}$ und damit die vorgeschlagenen Formulierungen ${ }^{4}$ der Verbindung VII d ${ }^{3}$ vom Schmp. $167^{\circ}$ als 2 - $(\alpha$-Acetamino- $\alpha$-hydroxymethyl- $\beta$-hydroxy-propionyl) -thiophen (V) und dessen Acetylierungsproduktes XVI d ${ }^{3}$ vom Schmp. $157^{\circ}$ als 2-( $\alpha$-Acetamino- $\alpha$ acetoxymethyl- $\beta$-acetoxy-propionyl) -thiophen (VII). Mangels Vorrat wurde das aus der Verbindung VII ${ }^{3}$ durch Salzsäure-Hydrolyse hergestellte, bei $200^{\circ}$ sich zersetzende Chlorhydrat nicht nachanalysiert $^{6}$.

Unsere Nachprüfung der verschiedenen Kondensationsbedingungen von 2-Acetaminoacetyl-thiophen (I) mit Formaldehyd bestätigte ferner, daß mit 1,2 Äquival. $\mathrm{HCHO}$ und $\mathrm{K}_{2} \mathrm{CO}_{3}{ }^{1}$ oder mit 1 Äquival.

${ }^{4}$ Vgl. R. Ettore u. A. Vecchi, Z. Naturforschg. 12 b, 479 [1957].

5 H. UlRICH, Dissertation, Erlangen 1952.

6 Bei weiterer Nachforschung stellte sich heraus, daß die seinerzeit analysierende Laborantin Analysenergebnisse gefälscht hat. 
$\mathrm{HCHO}$ und $\mathrm{NaHCO}_{3}+\mathrm{Na}_{2} \mathrm{CO}_{3}{ }^{2}$ oder mit 1,9 Äquival. HCHO und $\mathrm{NaHCO}_{3}{ }^{3}$ ganz überwiegend das einfache, bei $113^{\circ}$ schmelzende Kondensationsprodukt III entsteht, während wir mit 3,5 Äquival. HCHO und $\mathrm{K}_{2} \mathrm{CO}_{3}$ ausschließlich das doppelte, bei $167^{\circ}$ schmelzende Kondensationsprodukt V gefaßt haben. Das frühere ${ }^{3}$, nicht mehr rekonstruierbare Kondensationsergebnis von $\mathrm{ULRICH}^{5}$ wäre verständlich, wenn etwa an Stelle von $\mathrm{NaHCO}_{3}$ das stärker alkalische $\mathrm{Na}_{2} \mathrm{CO}_{3}$ oder $\mathrm{K}_{2} \mathrm{CO}_{3}$ verwandt worden wäre.

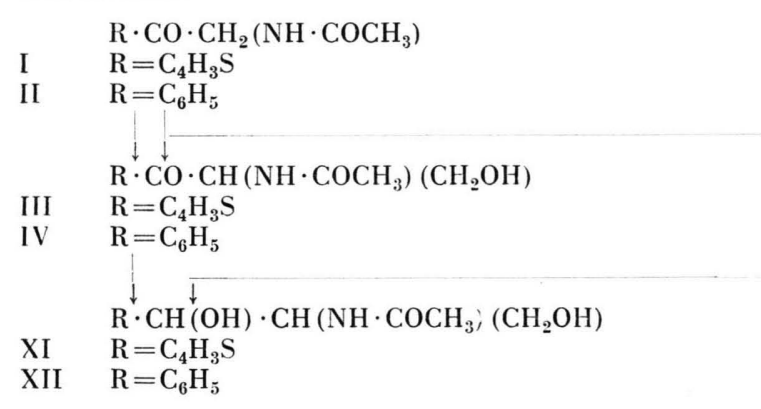

Schließlich hatten wir noch das Reduktionsverhalten der doppelten Kondensationsprodukte V und VI nachzuprüfen. VI wurde in Äthanol mit $\mathrm{Pd}+\mathrm{H}_{2}$ glatt hydriert zu X, welches auch bei der $\mathrm{NaBH}_{4}$ Reduktion von VI in Methanol entstand. Bei der Meerwein-Ponndorf-Reduktion mit Aluminiumisopropylat in Isopropanol jedoch ließ sich als einziges Hydierungsprodukt das bekannte DL-threo1-Phenyl-2-acetamino-propandiol-(1.3) (XII) ${ }^{8}$ fassen, wobei der vermutlich in einer Gleichgewichtsreaktion abgespaltene Formaldehyd sowohl im Destillat als auch im Reduktionsansatz nachgewiesen werden konnte; somit darf man annehmen, daß auch umgekehrt die Formaldehyd-Kondensation von Acetaminomethyl-aryl-ketonen mit Alkoholaten gelingen sollte. Etwas weniger vollständig erfolgte die Formaldehydabspaltung bei der Meerwein Ponndorf-Reduktion der Thiophenverbindung $\mathrm{V}$; zwar bestand das Reduktionsgemisch auch hier überwiegend aus dem bei $144^{\circ}$ schmelzenden, unter Formaldehydabspaltung entstandenen, bekannten DL-

7 M. C. Rebstock u. E. L. Pfeiffer, J. Amer. chem. Soc. 74, 3207 [1952].

8 L. M. Long u. H. D. Troutman, J. Amer. chem. Soc. 71, 2472 [1948].
Nachdem wir aus $\omega$-Acetamino-acetophenon (II) mit 4 Äquival. $\mathrm{HCHO}$ und $\mathrm{K}_{2} \mathrm{CO}_{3}$ ebenfalls glatt ein doppeltes Kondensationsprodukt VI erhalten haben, ergibt sich im Verein mit Befunden von Rевsтоск und Pfeiffer ${ }^{7}$, daß die stufenweise Anlagerung von zwei Moll. Formaldehyd an Acetaminomethyl-arylketone begünstigt wird durch Formaldehydüberschuß, durch stark basische Katalysatoren, Alkalicarbonat an Stelle von $\mathrm{NaHCO}_{3}$, durch erhöhte Reaktionstemperaturen und durch verlängerte Reaktionszeiten.

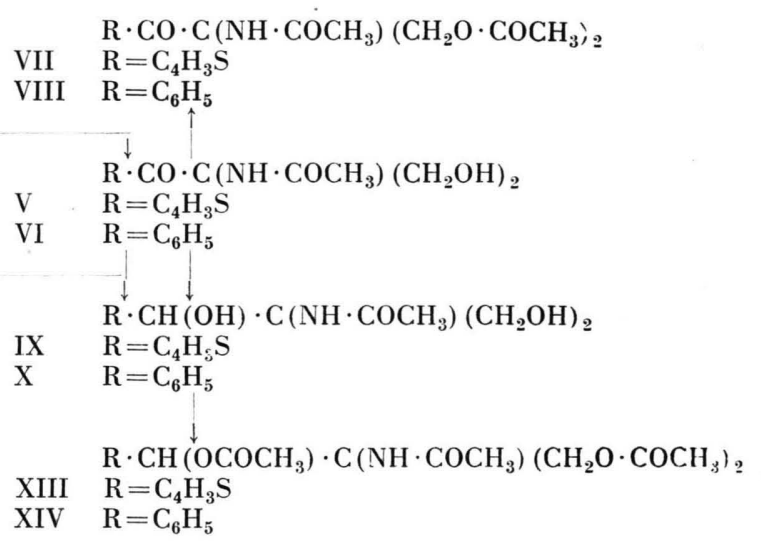

threo- 1 - [Thienyl- $\left(2^{\prime}\right)$ ] - 2-acetamino-propandiol-1.3 (XI) ${ }^{1,2}$, aber daneben ließ sich gelegentlich im K o fle r - Heiztischmikroskop die bei $132^{\circ}$ schmelzende Verbindung IX erkennen, deren Abtrennung durch fraktionierte Kristallisation $\mathrm{ULRICH}^{5}$ in einem Falle geglückt war, und die wiederum auf Grund falscher Analysen ${ }^{6}$ seinerzeit irrtümlicherweise als erythro-Racemat (VIII d ${ }^{3}$ ) angesehen worden war. In Wirklichkeit handelt es sich bei dem Reduktionsprodukt vom Schmp. $132^{\circ}$ um das ohne Formaldehydabspaltung entstandene DL-1-[Thienyl- $\left.\left(2^{\prime}\right)\right]-2$ acetamino-2-hydroxymethyl-propandiol-(1.3) (IX), welches durch $\mathrm{NaBH}_{4}$-Reduktion von $\mathrm{V}$ unschwer zugänglich ist.

\section{Beschreibung der Versuche*}

$2-(\alpha-\mathrm{A}$ c e t a mino $-\alpha-\mathrm{h}$ yd rox y meth y $l-\beta$ hydroxy-propionyl)-thiophen (V) ${ }^{4}$

$14 \mathrm{~g}(77 \mathrm{mMol})$ 2-(Acetaminoacetyl)-thiophen und $8 \mathrm{~g}(265 \mathrm{mMol})$ Paraformaldehyd wurden in $50 \mathrm{ml}$ Wasser unter gutes Rühren suspendiert und im Wasser-

* Die aufgeführten Mikroanalysen wurden bei A. Bernhardt, Mülheim (Ruhr), angefertigt. Die Schmelzpunktsangaben sind nicht korrigiert. 
bad bei langsam ansteigender Temperatur erwärmt. Bei $35^{\circ}$ wurde $1 \mathrm{~g} \quad(7,3 \mathrm{mMol})$ Kaliumcarbonat zugesetzt und die Temperatur langsam auf $45^{\circ}$ gesteigert. In wenigen Min. erfolgte Auflösung der suspendierten Substanz mit bräunlicher Farbe. Nach 15 Min. wurde die noch warme Lösung filtriert und im Flüssigkeitsextraktor mit $200 \mathrm{ml}$ Essigester unter Rückfluß $10 \mathrm{Stdn}$. lang extrahiert. Nach jeweils $2 \mathrm{Stdn}$. unterbrach man die Extraktion, um ausgefallene Substanz abzufiltrieren. Es fielen so insgesamt $10,2 \mathrm{~g}(55 \% \mathrm{~d}$. Th.) $\mathrm{V}$ an. Aus Essigester, 2-mal ungelöst, erhielt man farblose Kristalle vom Schmp. $167^{\circ}$, die in Methanol und Äthanol leicht, in Wasser weniger leicht, in Benzol, Chloroform und Äther schwer löslich waren.

$\mathrm{C}_{10} \mathrm{H}_{13} \mathrm{O}_{4} \mathrm{NS}(243,3) \quad$ Ber. C 49,37 H 5,38 N 5,76.

Gef. C 49,44 H 5,35 N 5,72.

$\alpha-\mathrm{A}$ ce t a mino- $\alpha-\mathrm{h}$ y d rox y meth y $\mathrm{l}-\beta$ hydroxy-propiophenon (VI)

$26,4 \mathrm{~g} \quad(150 \mathrm{mMol}) \quad \omega$-Acetamino-acetophenon und $18 \mathrm{~g}(600 \mathrm{mMol})$ Paraformaldehyd wurden in $100 \mathrm{ml}$ Wasser unter kräftigem Rühren suspendiert und im Wasserbad bei langsam ansteigender Temperatur erwärmt. Bei $35^{\circ}$ setzte man $1,2 \mathrm{~g}(8,7 \mathrm{mMol})$ Kaliumcarbonat zu, worauf in wenigen Min. eine klare, bräunliche Lösung entstand. Dabei war die Temperatur auf $45^{\circ}$ gestiegen. Ohne weitere Erwärmung wurde das langsam sich abkühlende Gemisch noch 45 Min. weitergerührt. Die filtrierte Lösung extrahierte man im Flüssigkeits-Extraktor unter Rückfluß $10 \mathrm{Stdn}$. mit $400 \mathrm{ml}$ Essigester und trocknete den Essigester mit Natriumsulfat. Beim Eindampfen auf ca. $75 \mathrm{ml}$ fielen $14 \mathrm{~g}$ vom Schmp. $135-138^{\circ}$ aus. Aus dem heißen Filtrat erhielt man beim Abkühlen weitere $4 \mathrm{~g}$ vom Schmp. 139 bis $141^{\circ}$ (51\% d. Th.). Durch einmaliges Umlösen aus Essigester mit wenig Äthanol stieg der Schmp. auf 140 bis $142^{\circ}$. VI löste sich leicht in Methanol und Äthanol, weniger leicht in Essigester, Amylacetat und Chloroform, schwer in Benzol, Äther und Ligroin.

$\mathrm{C}_{12} \mathrm{H}_{15} \mathrm{O}_{4} \mathrm{~N}(237,3) \quad$ Ber. N 5,90.

Gef. N 5,74.

$\alpha-A c e t a m i n o-\alpha-a c e t o x$ y met h y $1-\beta$ a cetoxy-propiophenon (VIII)

$0,5 \mathrm{~g}$ (ca. $2 \mathrm{mMol}$ ) VI wurden in $2 \mathrm{ml}$ trockenem Pyridin gelöst und, nach Abkühlung auf $0^{\circ}$, mit $1 \mathrm{ml}$ (10 mMol) Acetanhydrid versetzt. Nach 14-stdg. Stehen bei Raumtemperatur setzte man $25 \mathrm{ml}$ Eiswasser $\mathrm{zu}$ und schüttelte 4-mal mit je $30 \mathrm{ml}$ Essigester aus. Der Essigester wurde mit 2-n. $\mathrm{H}_{2} \mathrm{SO}_{4}, \mathrm{NaHCO}_{3}$-Lösg. und Wasser gewaschen, mit Natriumsulfat getrocknet und danach i. V. bis auf $5 \mathrm{ml}$ abgezogen. Es fielen $250 \mathrm{mg}$ VIII (39\% d. Th.) in farblosen Kristallen vom Schmp. $125-127^{\circ}$ aus. Aus wenig Essigester umgelöst betrug der Schmp. $126-127^{\circ}$.

$\mathrm{C}_{16} \mathrm{H}_{19} \mathrm{O}_{6} \mathrm{~N}(321,3) \quad$ Ber. $\mathrm{N} 4,36$.

Gef. N 4,37.
DL - 1 - Phenyl-2 - a c e t a mino-2 -

hydrox y met h y l-propandiol-(1.3) (X)

a) Reduktion mit Wasserstoff und $\mathrm{PdO}$

$1 \mathrm{~g} \mathrm{PdO}^{9}$ (82 mMol) wurde in $60 \mathrm{ml}$ abs. Äthanol bei Raumtemperatur mit Wasserstoff geschüttelt bis keine Aufnahme mehr erfolgte. Nach Evakuierung der Schüttelente ließ man $6 \mathrm{~g}(25 \mathrm{mMol}) \mathrm{VI}$, gelöst in $80 \mathrm{ml}$ warmem abs. Äthanol, einlaufen und schüttelte mit Wasserstoff bei Raumtemperatur. Nach 8 Stdn. war die theor. Menge $(600 \mathrm{ml} \mathrm{H})$ aufgenommen, die sich in 2 weiteren Stdn. nicht vermehrte. Man filtrierte die Lösung und engte i. V. bei $30^{\circ}$ stark ein. Nach 2 Tagen waren aus der öligen Lösung $5,2 \mathrm{~g}$ ( $86 \%$ d. Th.) $\mathrm{X}$ in farblosen Kristallen gefallen, die aus Essigester mit dem Schmp. $103-104^{\circ}$ sich abschieden.

$\mathrm{C}_{12} \mathrm{H}_{17} \mathrm{O}_{4} \mathrm{~N}(239,3) \quad$ Ber. N 5,85.

Gef. N 5,97.

\section{b) Reduktion mit $\mathrm{NaBH}_{4}$}

$0,5 \mathrm{~g}$ (ca. $2 \mathrm{mMol}$ ) VI wurden in $10 \mathrm{ml}$ wasserfreiem Methanol gelöst, $50 \mathrm{mg}$ (ca. 1,2 mMol) $\mathrm{NaBH}_{4}$ zugesetzt und im Wasserbad 15 Min. bei leichtem Sieden gehalten. Nach kurzem Stehen säuerte man mit 2-n. $\mathrm{H}_{2} \mathrm{SO}_{4}$ an $\left(p_{\mathrm{H}} 3-4\right)$ und erhitzte nochmals $15 \mathrm{Min}$. zum schwachen Sieden. Nach 1-stdg. Stehen wurde von ausgefallenem Natriumsulfat abfiltriert und die Lösung i. V. eingedampft. Den zähen, öligen Rückstand nahm man mit einigen $\mathrm{ml}$ heißem Essigester auf und trocknete mit Natriumsulfat. Nach Einengen der filtrierten Lösung bis auf $3 \mathrm{ml}$ versetzte man in der Hitze mit einigen Tropfen Äthanol und ließ abkühlen. Es kristallisierten nach längerem Stehen $150 \mathrm{mg}$ X (30\% d. Th.) vom Schmp. $102-103^{\circ}$ aus, welches im Gemisch mit dem obigen Produkt unverändert schmolz.

D L $-N, O, O, O-\mathrm{T}$ e t r a a c e t y $\mathrm{l}-1-\mathrm{p}$ he n y $\mathrm{l}-2$ a mino-2-hy d rox y methyl-p ropandiol-

(1.3) (XIV)

$0,5 \mathrm{~g}$ (ca. $2 \mathrm{mMol}$ ) $\mathrm{X}$ wurden in $5 \mathrm{ml}$ trockenem $\mathrm{Py}$ ridin mit $2 \mathrm{ml}(20 \mathrm{mMol})$ Acetanhydrid acetyliert. Nach 2 Tagen goß man die Lösung in $50 \mathrm{ml}$ Eiswasser und schüttelte mit Essigester aus. Die mit 2-n. $\mathrm{H}_{2} \mathrm{SO}_{4}$, $\mathrm{NaHCO}_{3}$-Lösg. und Wasser gewaschene Lösung wurde i. V. eingeengt. Man erhielt XIV in farblosen Kristallen, die bei $101-102^{\circ}$ schmolzen. Aus Essigester umgelöst stieg der Schmp. auf $101-102,5^{\circ}$.

$$
\begin{array}{ll}
\mathrm{C}_{18} \mathrm{H}_{23} \mathrm{O}_{7} \mathrm{~N}(365,4) & \text { Ber. N 3,83. } \\
& \text { Gef. N 3,96. }
\end{array}
$$

DL - t h reo-1-Ph en y l-2 - a c e t a minop r o p a n dio $1-(1.3)^{8}$ (XII)

$4,75 \mathrm{~g}$ (20 mMol) VI wurden in $80 \mathrm{ml}$ Isopropanol gelöst und $4 \mathrm{~g}(20 \mathrm{mMol})$ Al-isopropylat in $80 \mathrm{ml}$ Isopropanol zugesetzt. Die Reduktion wurde bei einer Badtemperatur von $115-120^{\circ}$ wie unten bei XI beschrieben durchgeführt. Nach 9 Stdn. hydrolysierte man

9 Org. Synthes. Coll. Vol. II, S. 566, New York 1943. 
mit $5 \mathrm{ml}$ Wasser durch $10 \mathrm{Min}$. langes Kochen unter Rückfluß. Dann wurde heiß abgenutscht und der Rückstand mit $100 \mathrm{ml}$ heißem, 80-proz. Isopropanol in Portionen extrahiert. Sowohl das Destillat als auch die Reduktionslösg. gaben Formaldehyd-Reaktion nach Schryver ${ }^{10}$ und nach Pilhazy ${ }^{10}$. Das Filtrat hinterließ beim Einengen i. V. ein gelbliches Öl, das in wenig heißem Essigester gelöst wurde und nach Stehenlassen im Eisschrank beim Anreiben 4,5 g klebrige Kristalle lieferte. Aus Amylacteat umgelöst, schmolzen sie bei 135 bis $136^{\circ}$; Die Mischung mit einem nach Long und Troutman 8 erhaltenen Produkt schmolz unverändert bei $135-136^{\circ}$. Kristallisierte Nebenprodukte waren nicht $\mathrm{zu}$ entdecken.

$$
\begin{gathered}
\text { DL - threo- } 1-\left[\text { Thien y } 1-\left(2^{\prime}\right)\right]-2-\text { a c e t a m in o- } \\
\text { propandio } 1-(1.3) \text { (XI) }
\end{gathered}
$$

$3 \mathrm{~g}$ (12 mMol) V wurden in $30 \mathrm{ml}$ Isopropanol gelöst und eine Lösung von $5 \mathrm{~g}(25 \mathrm{mMol})$ Al-isopropylat in $60 \mathrm{ml}$ Isopropanol zugesetzt. Bei einer Badtemperatur von $120-130^{\circ}$ wurde ein Aceton-IsopropanolGemisch über eine V i g r e u x - Kolonne $(30 \mathrm{~cm}) \mathrm{mit}$ eingesetztem Dephlegmator langsam abdestilliert. Die abdestillierte Menge wurde durch Zutropfen von Isopropanol zum Reduktionsgemisch laufend ergänzt. Nach $10 \mathrm{Stdn}$. war mit 2.4-Dinitrophenylhydrazin kein Aceton mehr nachweisbar. Die Reduktionslösung wurde nach Zusatz von $8 \mathrm{ml}$ Wasser 15 Min. unter Rückfluß erhitzt, dann noch heiß abgenutscht und der Rückstand mit 30, $30,20 \mathrm{ml}$ heißem, 80-proz. Isopropanol, dann 2-maỉ mit je $20 \mathrm{ml}$ heißem Isopropanol extrahiert. Sowohl im Destillat als auch in der Reduktionslösung war HCHO nach Schryver ${ }^{*} 10$ und nach Pilhazy * 10 nachweisbar.

Die Reduktionslösung wurde bei einer Badtempera. tur bis $70^{\circ}$ i. V. eingedampft. Man erhielt $4 \mathrm{~g}$ einer braunen, öligen Flüssigkeit, die mit $7 \mathrm{ml}$ heißem Essigester aufgenommen wurde. Beim Abkühlen fielen

* Nachweis von Schryver ${ }^{10}: 10 \mathrm{ml}$ Analysenlösung $+2 \mathrm{ml}$ 1-proz. salzsaurer Phenylhydrazinlösg. $+1 \mathrm{ml}$ 5-proz. $\mathrm{K}_{4} \mathrm{Fe}(\mathrm{CN})_{6}$-Lösg. $+5 \mathrm{ml}$ konz. $\mathrm{HCl}$ : fuchsinähnliche $\mathrm{Fär}$ bung.
$350 \mathrm{mg}$ Reduktionsprodukt in farblosen Kristallen vom Schmp. $127-131^{\circ}$ aus. Aus der Mutterlauge gewann man weitere $250 \mathrm{mg}$. Beim weiteren Einengen erhielt man ein gelbes, nach Akazien riechendes Ö̉. Die aus Essigester umgelösten, farblosen Kristalle schmolzen bei $128-131^{\circ}$. Unter dem Kofler-Heiztischmikroskop waren 2 Kristallformen zu erkennen: Prismen, die um $133-134^{\circ}$ schmolzen, IX, und Nadeln, die um $139-141^{\circ}$ schmolzen, XI. $150 \mathrm{mg}$ des farblosen Kristallisates ergaben nach dem Umlösen aus 1,5 ml Essigester wiederum ein Gemisch.

Bei der Mehrzahl der Ansätze fiel beim Umlösen des kristallisierten Reduktionsproduktes nur bei 141 bis $142^{\circ}$ schmelzendes XI ${ }^{1,2}$ an.

D L - $1-\left[\right.$ T h i en y l- $\left.\left(2^{\prime}\right)\right]-2$ - a c e t a m in o- 2 hydroxymet hyl-propandiol-(1.3) (IX)

$0,5 \mathrm{~g}$ (2 mMol) $\mathrm{V}$ wurden in $10 \mathrm{ml}$ abs. Methanol gelöst, mit $50 \mathrm{mg}(1,3 \mathrm{mMol}) \mathrm{NaBH}_{4}$ versetzt und das Gemisch im Wasserbad 2 Stdn. bei schwachem Sieden gehalten. Dann säuerte man mit 2-n. $\mathrm{H}_{2} \mathrm{SO}_{4}$ schwach an $\left(p_{\mathrm{H}} 3-4\right)$ und erhitzte weitere $15 \mathrm{Min}$. gelinde, um gebildete, recht beständige Borsäureester zu zerlegen. Das ausgefallene Natriumsulfat wurde abfiltriert und das Filtrat i. V. eingedampft. Den zähen, öligen Rückstand nahm man mit einigen ml heißem Essigester auf, trocknete mit $\mathrm{Na}_{2} \mathrm{SO}_{4}$ und dampfte das Lösungsmittel bis auf $1 \mathrm{ml}$ ein. Nach Zusatz von einigen Tropfen Äthanol in der Siedehitze kristallisierten beim Abkühlen $250 \mathrm{mg}$ ( $50 \%$ d. Th.) vom Schmp. $129-130^{\circ}$ aus. Das aus Essigester umgelöste IX schmolz bei 130 bis $132^{\circ}$. Die Verbindung ist in Äthanol, Methanol, Aceton und Wasser leicht, in kaltem Essigester schwer löslich, in Benzol, Chloroform fast unlöslich.

$\mathrm{C}_{10} \mathrm{H}_{15} \mathrm{O}_{4} \mathrm{NS}(245,3) \quad$ Ber. C 48,96 H 6,16 N 5,71. Gef. C 49,13 H 6,36 N 5,49.

** Nachweis nach Pilhazy ${ }^{10}$ : $15 \mathrm{ml}$ Analysenlösg. $+1 \mathrm{ml}$ 1-proz. salzsaurer Phenylhydrazinlösg. + einige Tropfen frisch bereiteter Natrium-nitroso-prussiat-Lösg. + einige Tropfen verd. $\mathrm{NaOH}$ : Blaufärbung.

10 Th. Sabalitschka u. Cl. Harnisch, Pharmaz. Zentralhalle Deutschland 67, 371 [1926]. 